\title{
Functionalization of Renewable Materials for Energy, Environment, and Healthcare
}

\author{
Rajan Jose \\ Nanostructured Renewable Energy Materials Laboratory, Universiti Malaysia Pahang, \\ 26300 Kuantan, Malaysia; \\ Correspondence: rjose@ump.edu.my
}

\begin{abstract}
Sustainability has emerged as a keyword in all aspects of life whether it is resources or technologies and products or processes. Besides, nearly a billion new consumers join the society in 13-15 years; and the growing demand for higher standards of living make the worldwide materials consumption continuously growing. Strategic solutions are therefore required not only for addressing the gaps but also for eliminating the undesirable environmental effects of supply chain to ensure quality and sustainable living. This lecture shares the experience in developing renewable materials for energy, environment, and healthcare to mitigate not only waste biomass but also to their value addition for viable products. Carbons and cellulose were produced from one of Malaysia's largest agriculture sector - the oil palm industry. Nearly a ton of waste biomass, including palm kernel shell and empty fruit bunches, is produced for every $25 \mathrm{~kg}$ of crude palm oil - their efficient utilization would promote small and medium scale industries. High quality activated carbons were produced from palm kernel shells and evaluated their usefulness in energy storage applications; the palm kernel shell activated carbons gave one of the best supercapacitive charge storage performances. Besides, the performance of the materials is increased to many folds when hierarchical ceramic nanostructures were filled in their passive pores. We have further discovered formation of thin metal and metal oxide films on at the palm kernel shell derived carbon surface, which has taken the supercapacitive charge storage to record values. Further, we have derived nanocellulose from empty fruit bunches which are developed into nonwoven fibrous films, using them as filter membranes and palm kernel shell carbon as adsorbents portable water filters have been produced - a portable device that would support ecotourism. The fibrous films along with natural antioxidents has also been demonstrated. The cellulose was found to be good dispersant as nanocoolants and so on. The lecture will also highlight development in composite materials from non-renewable sources for solar energy conversion and charge storage devices.
\end{abstract}

